\title{
reingerfión de polvo en hornos rotatorios por vía húmeda
}

JULIO FERNANDEZ VAZQUEZ-GULIAS

Lcdo. en Ciencias Químicas

\section{ANTECEDENTES}

Es bien sabido la enorme ventaja económica que representa el aprovechamiento del polvo que, en un porcentaje superior al $5 \%$ de ia producción total de clínker, sale arrastrado con ios gases de los hornos rotatorios.

Aprovechar este polvo es de gran interés técnico-económico, porque supone el consiguiente incremento de producción al ser transformado en clínker.

En alguna planta se logró, después de varios ensayos, efectuar la reinyección por un tubo paralelo al quemador.

También se hicieron otras pruebas tratando de alimentar el polvo mezclado con distintos porcentajes de agua, sin obtener buenos resultados.

Asimismo, tenemos noticias de otros intentos que se han hecho para aprovechar este polvo introduciéndolo (por aberturas sobre la chapa del horno) mediante sistemas más o menos complicados, y también, por insuflación y mezcla con el combustible.

A pesar de todo ello, la solución de este aprovechamiento presenta muchos problemas, tanto por la complejidad de los métodos empleados como por la serie de dificultades que se originan en la marcha regular de los hornos, llegando, incluso, a desecharse para evitar estos inconvenientes.

\section{REINYECCION}

La idea del autor partió de un enfoque sencillo de la cuestión: tratar de aprovechar el polvo seco (como sale de los hornos) utilizando la pasta que pasa por el tubo de alimentación como vehículo de transporte.

Habíamos pensado «a priori» que:

1) La adición de polvo iba a suponer un incremento de la viscosidad. 
2) Este incremento de viscosidad debería ser función de:
a) la composición química del polvo (el cual está parcialmente calcinado);
b) la granulometría del material (con un porcentaje aumentado de partículas finas);
c) el tiempo que permanece en contacto (homogeneización del polvo con la pasta).

3) Este mismo aumento de viscosidad iba a favorecer la formación de nódulos, así como lograr que tuvieran más resistencias.

4) No se espera tener dificultades en las reacciones químicas del proceso de cocción.

5) Los inconvenientes a temer eran derivad os del carácter hidráulico del mismo polvo:

a) obstrucción de los tubos de alimentación: polvo y pasta;

b) posible formación de un anillo de pasta en la entrada del horno.

\section{- PRUEBAS DE LABORATORIO}

se han comprobado experimentalmente los apartes 1) y 2) con los materiales preparados de acuerdo con el siguiente cuadro:

\section{MATERIAL}

Pasta cruda (sin adición de polvo)

" $\quad+3 \%$ polvo (después de $10 \mathrm{~s}$ de agitación manual)

$" \quad+3 \% "$ (después de $60 \mathrm{~s}$ de agitación mecánica) $\ldots \ldots \ldots \ldots \ldots \ldots$

$" \quad+6 \%$ (después de $10 \mathrm{~s}$ de agitación manual) $\ldots \ldots \ldots \ldots \ldots \ldots \ldots$

, $\quad+6 \%$ (después de $60 \mathrm{~s}$ de agitación mecánica) $\ldots \ldots \ldots \ldots \ldots \ldots$

" $\quad+9 \% \quad$ (después de $10 \mathrm{~s}$ de agitación manual) $\ldots \ldots \ldots \ldots \ldots \ldots \ldots$

$" \quad+9 \% \quad$ (después de $60 \mathrm{~s}$ de agitación mecánica) $\ldots \ldots \ldots \ldots \ldots \ldots$

i c composición química del polvo es la siguıente:

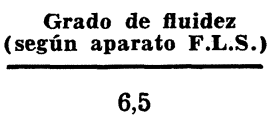

5,0

2,25

3,0

1,75

1,75

1,25

$\begin{array}{llr}\mathrm{SiO}_{2} & : & 17,08 \% \\ \mathrm{Al}_{2} \mathrm{O}_{3} & : & 3,39 " \\ \mathrm{Fe}_{2} \mathrm{O}_{3} & : & 2,23 " \\ \mathrm{CO}_{2} & : & 20,34 " \\ \mathrm{CaCO} & : & 46,25 " \text { (calculado a partir del } \mathrm{CO}_{2} \text { ) } \\ \mathrm{CaO} & : & 53,40 " \text { (un 25,91 \% corresponde al carbonat, } \\ \mathrm{MgO} & : & 0,64 " \\ \mathrm{Na} \mathrm{O}_{2} \mathrm{O} & : & 0,84 " \\ \mathrm{~K} \mathrm{O} & : & 0,99 " \\ \mathrm{Ms} . & : & 3,04 " \\ \mathrm{Mf} . & : & 1,52 \\ \mathrm{Mh} . & : & 2,35 \\ \mathrm{Sat} . & : & 100,5 "\end{array}$

$46,25 / 76,40 \simeq 60 \%$ de material sin calcinar.

Nota: 76,40 era el carbonato valorado en un c:udo empleado el dia del ensayc 
El análisis granulométrico es el siguiente:

\begin{tabular}{cr} 
T a & m i z : \\
\hline n.o & 30 \\
n.o & 50 \\
n.o & 70 \\
n.o & 100 \\
n.o & 200 \\
n. & 200
\end{tabular}
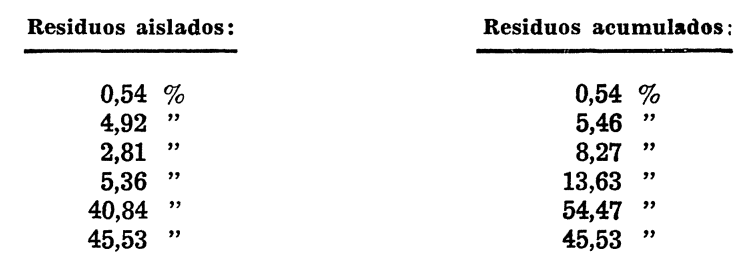

Queremos observar que la adición de este polvo apenas influye sobre la granulometría de la pasta, toda vez que, aunque aparece un 54,5\% sobre el tamiz n. ${ }^{\circ} 200$, estos valores son aparentes por surgir aglomeraciones de partículäs finas.

Otra condición favorable se reflejó en las pruebas de aumento de temperatura originada al mezclar el polvo con la pasta cruda.

Se hicieron tres ensayos poniendo pasta en una mezcladora mecánica y añadiéndole polvo (ambos a temperatura ambiente).

Los resultados fueron:
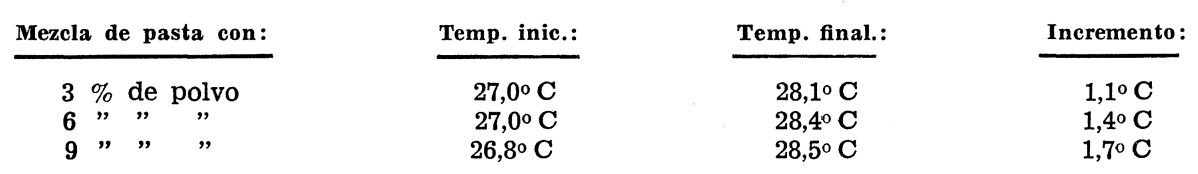

Para la mezcla de 250 c. c. de agua y $200 \mathrm{~g}$ de polvo, el incremento fue $2,5^{\circ} \mathrm{C}$.

\section{PRIMER ENSAYO:}

El día 19-10-65 se hizo la prueba inicial con un tubo de 1,5 m de longitud y 2" de $\varnothing$; alimentado por una pequeña rosca transportadora de velocidad variable.

Durante 1 hora se introdujeron por el tubo $1.000 \mathrm{~kg}$ de polvo (aprox. $4 \%$ de la producción real de clínker del horno).

El ensayo resultó totalmente satisfactorio, ya que en ningún momento hubo dificultades en la alimentación.

Con esta inyección (que podríamos llamar interna) se ha tratado de que la pasta no esté mucho tiempo en contacto con el polvo, con objeto de evitar endurecimientos prematuros.

Al comprobar los tubos de alimentación - pasta y polvo- se pudo notar que se encontraban perfectamente limpios, sin huellas de aglomeraciones fuera de lo normal.

Animados con esta prueba, el día 20-10-65 se 11ició el segundo ensayo. 


\section{-5 SEGUNDO ENSAYO:}

A las 10 de la mañana se comenzó la alimentación del polvo —después de haber limpiado con anterioridad el tubo de pasta- (lo cual se hace normalmente cada 2 horas). Se utilizó un tubo de $5 \mathrm{~m}$ de longitud y 2" de $\varnothing$ para la inyección del polvo (véase croquis adjunto).

La cantidad de polvo inyectada fue de $1.350 \mathrm{~kg} / \mathrm{hr}$ (aprox. $5 \%$ de la producción real de clínker).

En estas condiciones se trabajó ininterrumpidamente hasta las 12 de la mañana. A esta hora sc verificó el estado de los tubos.

Observamos que el tubo inyector de polvo estaba limpio y que el de pasta también se encontraba en buenas condiciones. Se procedió a la limpieza total del tubo de pasta y a los 5 minutos se volvió a introducir el polvo.

Así se continuó hasta las 2 de la tarde, sin novedad.

Al igual que la vez pasada, ambos tubos estaban en buenas condiciones al ser inspeccionados.

i a prueba se continuó entonces con el tubo de 1,5 m de longitud, para observar cualquier posible diferencia. A las 2-30 de la tarde se retiró el tubo de 1,5 m, pues se obstruyó; y en su lugar se puso el de $5 \mathrm{~m}$, con el cual se tráiajó hasta las 4 de la tarde.

Observados nuevamente los tubos se hallaron en buen estado y se volvió a colocar el de $5 \mathrm{~m}$ hasta las 4-30 de la tarde, hora en que se dio por terminada la prueba.

La alimentación de pasta se paró a las 5 de la tarde, y el horno, poco después, según estaba programado.

La obstrucción en el tubo de polvo no constituye obstáculo negativo para la prueba, ya que se produjo como consecuencia de haber rebajado la marcha del horno (el cual acusaba el excedente de carga).

Al ser menor la velocidad del horno disminuyó la entrada de pasta. Como no se rebajó la entrada de polvo fue mucho mayor la concentración de éste y, por lo tanto, se aumentó la viscosidad.

Debe señalarse que el tubo de pasta no se obstruyó en ningún momento.

i'or otra parte, estos inconvenientes sirvieron para concluir sobre una interesante observación: la cantidad de polvo a inyectar debe sincronizarse con la alimentación de la pasta.

insistimos que en condiciones normales no debe obstruirse el tuyo inyector de polvo, ya que existen principalmente tres fuerzas que se oponen a ello: 1) la gravedad; 2) la succión originada por el ventilador de tiro, y 3) el arrastre debido a la velocidad de la pasta que se desliza externamente a este tubo.

\section{DATOS SOBRE LA MARCHA DEL HORNO}

Debido al escaso porcentaje de agua en la pasta $(34,8 \%)$, el horno $\mathrm{n}^{\circ} 3(3,45 \mathrm{~m} \varnothing \times$ $\times 140 \mathrm{~m}$ ) estaba produciendo más de $620 \mathrm{t} /$ día, valor considerado como límite.

No se estimó conveniente rebajar la producción al inyectarle polvo, para observar las modificaciones que pudieran presentarse, y comprobar el acuse de exceso de carga, lo cual sería un justificativo más de que el polvo se estaba aprovechando. 


\section{CROQUIS REINYECCION POLVO}

\section{HORNOS ROTATORIOS}



1.-Plataforma sección alimentación. 2.-Entrada de pasta cruda. 3.-Tubo de alimentación de pasta. 4. -Entrada de polvo. 5.-Tubo de reinyección de polvo. 6.- Salida de polvo. 7.-Salida de la mezcla pasta-polvo. 8.- Variante eventual entrada polvo. 9.-Tubo guia. 10.-Perno de sujeción. 11.- Pared cámara de humos. 12.-Ladrillos refractarios horno. 13.-Chapa horno. 
Un dato muy interesante resultó ser el aumento de temperatura que acusó la cámara de humos 1 hora después de comenzado el ensayo.

Como consecuencia de este incremento hubo que cerrar la válvula de tiro, de acuerdo con el siguiente cuadro:

Hora del día:

Temp. cámara de humos $\left({ }^{\circ} \mathbf{C}\right)$ :

Válvula de tiro. Grados abertura:

Presión gas primario (libras/pulg²):

\begin{tabular}{rrrrrrrr}
$\mathbf{7} \mathbf{h r}$ & $8 \mathbf{h r}$ & $9 \mathbf{h r}$ & $10 \mathbf{h r}$ & $11 \mathbf{h r}$ & $12 \mathbf{h r}$ & $13 \mathbf{h r}$ & $14 \mathbf{h r}$ \\
\hline 320 & 325 & 320 & 320 & 330 & 325 & 330 & 330 \\
24 & 24 & 24 & 24 & 22 & 22 & 20 & 20 \\
8 & 7 & 9 & 8 & 8 & 8 & 7 & 8
\end{tabular}

Puede notarse la continuidad de la presión del gas, a pesar de lo cual se originó el fenómeno citado.

Aparentemente esto parece una contradicción, ya que al no rebajar la entrada de pasta, y por otra parte al añadirle polvo, era de esperar un descenso en la temperatura de la cámara de humos.

La explicación del fenómeno se debe encontrar en que, además de la mezcla física del polvillo con la pasta, se originan reacciones químicas de hidratación de la cal viva (principalmente) e, incluso, de posibles compuestos potenciales existentes en el polvo.

En estas reacciones exotérmicas creemos reside la explicación, ya que, además de engenArar calor, se rebaja (más de lo que era de esperar por cálculos aritméticos) el \% de agua debido a este mismo proceso de hidratación.

Esta circunstancia viene a ser otra de las cualidades favorables para recomendar el método utilizado.

Se observaron los porcentajes de agua en nódulos que resultaron normales. La consistencia de los mismos fue considerada mayor que de ordinario.

La parada del horno estaba prevista para revisar en los enfriadores, y sirvió para comprobar el interior del mismo.

Comenzando por el tubo de alimentación apareció — como es normal en estas paradas- con un recubrimiento de pasta debido a las salpicaduras.

Para comprobar la naturaleza de este recubrimiento se valoró una muestra exterior del mismo. Resultó ser análoga a la última alimentación de pasta cruda; lo cual excluye haya habido acumulación de polvo, porque este último es mucho menos rico en cabonatos.

Asimismo, pudimos observar que, tanto en la zona de cadenas como en la parte próxima a la misma, no se encontró muestra de formación de anillos de polvo.

Con estas observaciones despejamos las dudas al respecto. 
El clínker producido hasta la parada del horno conservó sus cualidades normales.

La expansión al autoclave (promedio del día del ensayo) para el horno $n .{ }^{\circ} 3$ fue de 0.08 por ciento.

RESUMEN

1) El aprovechamiento del polvo que sale con los gases de escape de los hornos rotatorios, por vía húmeda, es factible mediante su reinyección.

2) Dejamos explicada la manera de efectuar esta reinyección por un tubo interior al tubo de alimentación principal.

En el esquema anexo se indica otra eventual variante al introducir el polvo por un tubo vertịcal que se conecta al tubo de alimentación principal.

3) El peso de este polvo viene a representar un $5 \%$ o más de la producción total de clínker.

4) Algunas de las ventajas de nuestro método además de las generales, son:

a) la sencillez del mismo;

b) el ahorro calorífico que supone la reacción exotérmica del polvillo con la pasta;

c) el descenso en el porcentaje de agua, más allá de lo esperado para una mezcla física, originado como consecuencia de la hidratación del polvillo;

d) la influencia beneficiosa de la anterior reacción para hacer más consistentes los nódulos.

Nota: Este trabajo ha sido objeto de una patente de invención registrada en España, bajo el título "Sistema e instalación para la recuperación de polvo en hornos de cemento", con el n. ${ }^{\circ} 334585$, el 16 de diciembre de 1966. 